\title{
Correction to: Adrenocortical incidentalomas and bone: from molecular insights to clinical perspectives
}

\author{
Barbara Altieri $\rrbracket^{1,2} \cdot$ Giovanna Muscogiuri $\mathbb{D}^{3} \cdot$ Stavroula A. Paschou $\varpi^{4} \cdot$ Andromachi Vryonidou $^{5}$. \\ Silvia Della Casa $\mathbb{C}^{2} \cdot$ Alfredo Pontecorvi $\mathbb{D}^{2} \cdot$ Martin Fassnacht $\mathbb{D}^{1} \cdot$ Cristina L. Ronchi $\mathbb{D}^{1,6} \cdot$ John Newell-Price $\mathbb{(}^{7}$
}

Published online: 10 September 2018

(c) Springer Science+Business Media, LLC, part of Springer Nature 2018

\section{Correction to: Endocrine}

https://doi.org/10.1007/s12020-018-1696-Z

The original version of this article unfortunately contained a mistake in Fig. 1. There is a typo in the word "osteoclastogenesis" and the word "activity" is missing in the same entity. It should be "osteoclastogenesis" instead of "osteoclestogenesis". The corrected figure is given below.

The original article has been corrected.

The original article can be found online at https://doi.org/10.1007/ s12020-018-1696-z.

Barbara Altieri

altieri.barbara@gmail.com

1 Division of Endocrinology and Diabetes, Department of Internal Medicine I, University Hospital, University of Wuerzburg,

Wuerzburg, Germany

2 Division of Endocrinology and Metabolic Diseases, Institute of Medical Pathology, Catholic University of the Sacred Heart, Rome, Italy

3 Department of Clinical Medicine and Surgery, University "Federico II", Naples, Italy

4 Division of Endocrinology and Diabetes, "Aghia Sophia" Hospital, Medical School, National and Kapodistrian University of Athens, Athens, Greece

5 Department of Endocrinology and Diabetes, Hellenic Red Cross Hospital, Athens, Greece

6 Institute of Metabolism and System Research, University of Birmingham, Birmingham, UK

7 Department of Oncology and Metabolism, University of Sheffield Medical School, Sheffield, UK 


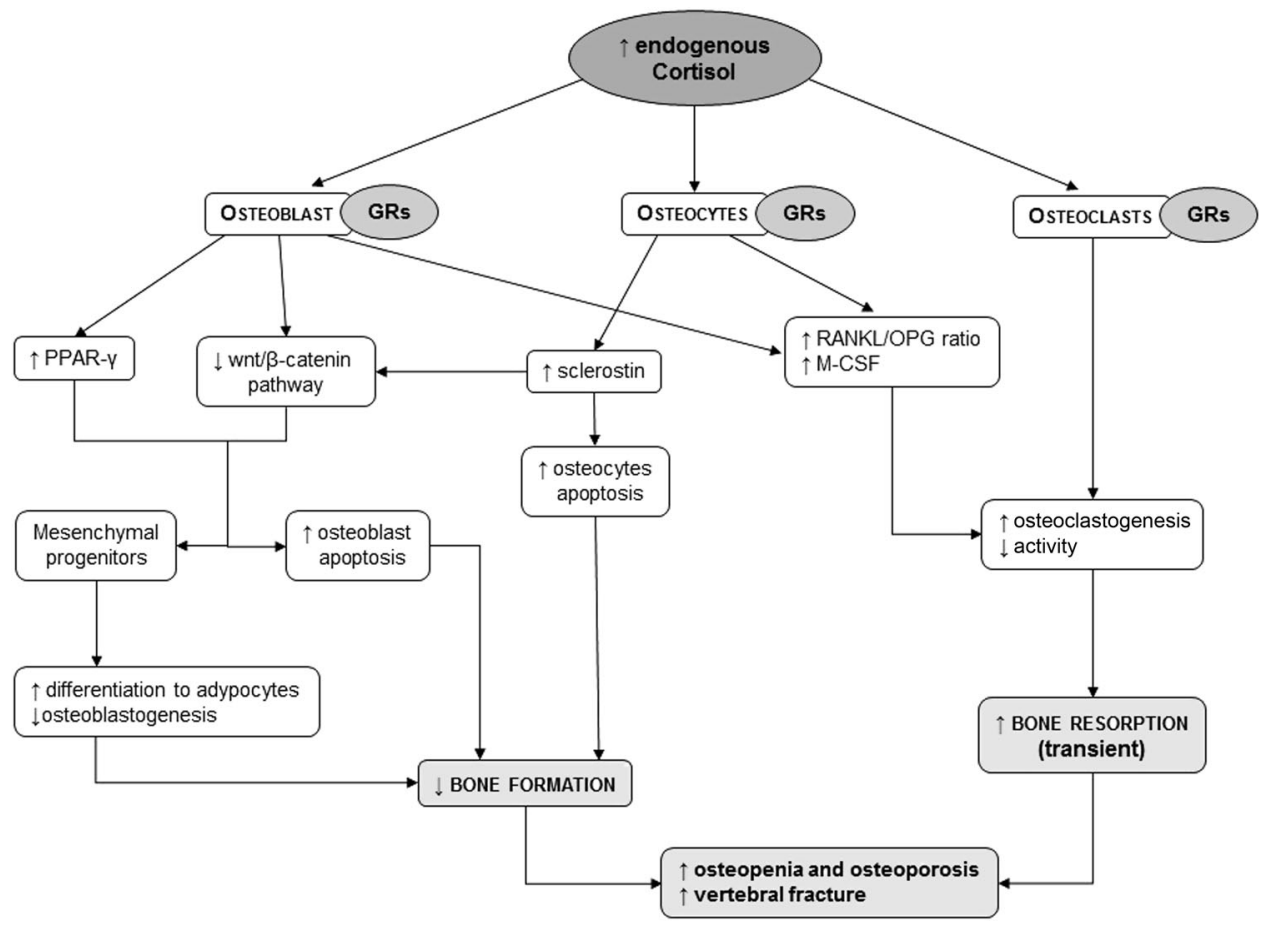

Fig. 1 Direct effects of cortisol excess on bone metabolism. Endogenous glucocorticoid excess negatively affect osteoblast, osteocytes, and osteoclast, which expressed glucocorticoid receptors (GRs). These actions include an upregulation of peroxisome proliferator-activated receptor (PPAR)- $\gamma$ [23] and an inhibition of the wingless (wnt)/ $\beta$ catenin signaling pathway [24-26], leading to mesenchymal progenitor cells differentiating preferentially into adipocyte that results in a decreased number of osteoblasts and in an increasing of osteoblast apoptosis and a consequent reduction of bone formation [28]. This mechanism is also stimulated by sclerostin produced by osteocytes [30]. Another key mechanism is the increase of the receptor activator for NF- $\mathrm{KB}$ ligand (RANKL)/osteoprotegerin (OPG) ratio produced by osteoblasts and osteocytes [32-34] that, together with the increased macrophage colony-stimulating factor (M-CSF) [36], stimulates osteoclastogenesis and bone resorption 